\title{
Accidental disgust does not cause moral condemnation of neutral actions
}

Jussi Jylkkäa Johanna Härkönen $^{\mathrm{b}}$, \& Jukka Hyönäb

aDepartment of Psychology, Abo Akademi University, Finland

Street address: Biskopsgatan 3, 20500 Turku, Finland

E-mail: jjylkka@abo.fi

${ }^{b}$ Department of Psychology, University of Turku, Finland

Street address: Assistentinkatu 7, 20500 Turku, Finland

E-mail: hyona@utu.fi 


\begin{abstract}
Emotivism in moral psychology holds that making moral judgements is at least partly an affective process. Three emotivist hypotheses can be distinguished: the elicitation hypothesis (that moral transgressions elicit emotions); the amplification hypothesis (that disgust amplifies moral judgments); and the moralization hypothesis (that affect moralizes the non-moral). Even though the moralization hypothesis is the strongest and most radical form of emotivism, it has not been systematically experimentally tested. Most previous studies have used as stimuli morally wrong actions, and thus they cannot answer whether disgust is sufficient to moralize an otherwise neutral action. In Experiment $1(N$ $=87)$ we tested the effect of accidental disgust on morally neutral scenarios, and in Experiment $2(N=$ 510) the differential effect of disgust on neutral and wrong scenarios. The results did not support either the moralization or the amplification hypothesis. Instead, Bayesian analyses provided substantial evidence for the null hypothesis that accidental disgust does not affect moral ratings. In line with recent literature, our study indicates that disgust is not a moralizing emotion. Data and materials are available at https://osf.io/wbyfu/
\end{abstract}

Keywords: Accidental Disgust; Moral Judgment; Moralization Hypothesis; Amplification Hypothesis; Moral Emotivism 


\section{Introduction}

Traditional theories of moral judgement (e.g., Kolhberg, 1981; Piaget, 1997) consider people as rational beings, who make moral judgements through rational reflection. They weigh the pros and cons of their actions, consider various rules that they should or should not follow, or even engage in highly abstract considerations such as what would happen if everyone would follow the same maxim as they do. Recent findings in moral psychology question this position, indicating that, at least in some cases such as sexual morality, people's moral judgements are not driven by rational reflection, but instead emotion. We may call moral psychological theories that posit some role for emotion in moral judgement emotivist theories of moral judgement. Following Avramova and Inbar (2013) and Landy and Goodwin (2015), we may distinguish between the three following emotivist claims:

(1) Emotions follow from moral judgements (the elicitation hypothesis)

(2) Emotions amplify moral judgements (the amplification hypothesis)

(3) Emotions moralise the non-moral (the moralization hypothesis)

The elicitation hypothesis is the most undisputed emotivist claim: for instance, reading about moral transgressions elicits facial expressions of disgust (Cannon, Schnall, \& White, 2011). There is also evidence for the amplification hypothesis: artificially induced disgust may amplify negative ratings of moral transgressions (e.g. Eskine, Kacinik, \& Prinz, 2011; Inbar, Pizarro, \& Bloom, 2011; Olatunji, Puncochar, \& Cox, 2016; Schnall, Haidt, Clore, \& Jordan, 2008; Ugazio, Lamm, \& Singer, 2012; Wheatley \& Haidt, 2005; but see Landy \& Goodwin, 2015). The moralization hypothesis, in turn, has received the least support, and is not directly addressed in the literature (Avramova \& Inbar, 2013; Landy \& Goodwin, 2015; Pizarro, Inbar, \& Helion, 2011). This is surprising, since the moralization 
hypothesis represents the strongest and most radical form of emotivism and is explicitly advocated by some researchers (e.g., Haidt, 2001; Prinz, 2006).

We will next review the previous studies addressing the role of emotions in moral judgment, focusing particularly on whether they support strong emotivism.

\section{Previous Research}

Correlational studies. The early studies on the role of emotion in moral judgement were interviews. For instance, Haidt, Koller, and Dias (1993) investigated how disrespectful but harmless actions are judged morally by participants of low and high socioeconomic status (SES) in the U.S. and Brazil. The scenarios depicted cleaning a toilet with one's national flag, consensual incest between siblings, or eating one's dead pet dog. They discovered that high-SES U.S. participants from elite universities considered the actions to be matters of social convention or personal preferences, whereas participants of low SES (particularly low-SES Brazilians) judged the actions to be immoral. Importantly, for those participants who judged the actions to be wrong, the judgement was better predicted by ratings of offensiveness than by ratings of harmfulness (which can be taken to reflect conceptual processes). On the contrary, for those participants who did not moralize the actions, the judgement was better predicted by ratings of harm than offensiveness (Haidt et al., 1993).

In a similar study, Haidt and Hersh (2001) interviewed American democrats and republicans about three kinds of sexual acts: homosexual sex, unusual forms of masturbation, and consensual incest between adult brother and sister. They discovered that republicans were less tolerant than democrats towards the actions, and that the republicans relied more on offensiveness than harm in justifying their answers. Regression analysis indicated that, in both groups, harm did not significantly predict moral condemnation in any of the scenarios, but instead affect and religious strength did, affect being the best predictor. The authors also discovered a phenomenon they entitled "moral dumbfounding", defined as 
“a confused inability to explain one's position” (Haidt \& Hersh 2001, p. 209), characterized by puzzlement, laughter, and stuttering (see also Haidt et al., unpublished manuscript). Importantly, however, Haidt and Hersh (2001) did not discover any moral dumbfounding in the classical Heinz dilemma (Kohlberg, 1981), whose ratings were best predicted by harm. ${ }^{1}$ It can be argued that the Heinz case was less emotional and more reflectively judged than the emotional scenarios.

There is also evidence that disgust sensitivity is associated with moral judgments (e.g. Chapman \& Anderson, 2014; Inbar, Pizarro, Knobe, \& Bloom, 2009; Jones \& Fitness, 2008). For instance, Chapman and Anderson (2014) presented evidence that participants with high disgust sensitivity rated moral transgressions more harshly. Relatedly, moral transgressions have been found to elicit facial expressions characteristic of disgust (Cannon et al., 2011; Chapman, Kim, Susskind, \& Anderson, 2009). Recent studies indicate, however, that disgust or disgust sensitivity is no more strongly associated with moral judgment than is sensitivity to other emotions (Landy \& Piazza, 2019). On the contrary, anger may be more consistently associated with moral condemnation than disgust (Piazza \& Landy, 2019).

All these studies are correlational and leave open the direction of causality — whether affect causes moral condemnation or vice versa. The moralization hypothesis is more directly assessed by

1 The Heinz dilemma is one of many fictitious scenarios used by Kohlberg (1981) to study the stages of moral development: "A woman was near death from a special kind of cancer. There was one drug that the doctors thought might save her. It was a form of radium that a druggist in the same town had recently discovered. The drug was expensive to make, but the druggist was charging ten times what the drug cost him to produce. He paid $\$ 200$ for the radium and charged $\$ 2,000$ for a small dose of the drug. The sick woman's husband, Heinz, went to everyone he knew to borrow the money, but he could only get together about $\$ 1,000$ which is half of what it cost. He told the druggist that his wife was dying and asked him to sell it cheaper or let him pay later. But the druggist said: 'No, I discovered the drug and I'm going to make money from it.' So Heinz got desperate and broke into the man's store to steal the drug for his wife. Should Heinz have broken into the laboratory to steal the drug for his wife? Why or why not?" (Kolhberg, 1981) 
experimental studies, which have typically focused on the effect of artificially induced disgust on moral ratings.

Experimental studies. Disgust has been discovered to amplify moral judgements (e.g., Eskine et al., 2011; Inbar et al., 2011; Olatunji et al., 2016; Schnall et al., 2008; Ugazio et al., 2012; Wheatley \& Haidt, 2005; but see Landy \& Goodwin, 2015). For instance, Eskine et al. (2011) induced disgust in a group of participants through making them drink a bitter tasting liquid. The disgust group made more severe moral ratings than controls in scenarios depicting moral transgressions, such as consensual incest or shoplifting. To take another example, Schnall et al. (2008) induced disgust in participants through bad smell, by making them conduct the test in a dirty office, recalling a disgusting experience, or through watching a disgusting video. In all conditions, the disgusted participants made more severe moral judgements about the described moral transgressions than the non-disgusted participants. The effect was mediated by the participants' sensitivity to feel disgust. A recent study by Tracy, Steckler, and Heltzel (2019), using a novel and creative setup, examined how administering an antiemetic (ginger) affects moral judgment. They found that ginger reduced the severity of judgments towards purity-based moral violations.

The previous studies are limited in two respects. First, they have mainly used scenarios depicting moral transgressions, and thus addressed only the amplification hypothesis and not the moralization hypothesis. That is, they have tested whether accidental disgust amplifies condemning judgments of actions that would have been judged as morally wrong already before the emotional induction. Thus, they cannot test the moralization hypothesis. Second, the studies have been plagued by small sample sizes and possibly a publication bias. A recent meta-analysis by Landy and Goodwin (2015) found only a very small amplifying effect of disgust $(d=.11)$, which disappeared completely after controlling for publication bias (but see Schnall, Haidt, Clore, \& Jordan, 2015), for a critical discussion). 
Only few studies have directly addressed the moralization hypothesis. Landy and Goodwin found evidence for a moralizing effect of disgust in their meta-analysis, but the effect was small $(d=$ $.21)$ and came from a small number of studies $(k=13)$. Moreover, there was evidence of a publication bias: all published studies $(k=6)$ supported a larger effect size than the unpublished studies $(k=7)$. To the best of our knowledge, no study has systematically studied the effect of disgust on morally neutral scenarios, and most of the evidence for the moralization hypothesis comes from individual cases (e.g., the student council case in Wheatley and Haidt, 2005). Systematically testing the moralization hypothesis is also motivated, because it is the strongest form of emotivism: it implies that disgust alone could moralize an otherwise neutral action.

\section{Aims and Hypotheses}

We tested the moralization hypothesis in two experiments. In Experiment 1, we tested whether disgust, artificially induced through viewing disgusting pictures, moralizes neutral actions, as predicted by the moralization hypothesis. In Experiment 2, we examined the differential effect of disgust, induced through viewing disgusting videos, on wrong and neutral scenarios. The moralization hypothesis implies that disgust would moralize neutral scenarios, but the amplification hypothesis predicts that disgust would only amplify judgments of morally wrong scenarios, without affecting judgments of neutral scenarios. To control for possible mediating variables, we also measured the political orientation (in both experiments) and disgust sensitivity (in Experiment 1) of the participants. Earlier research suggests that conservatives are more sensitive to disgust than liberals, and that disgust sensitivity may be associated with moral ratings (e.g., Haidt \& Hersh, 2001; Inbar \& Pizarro, 2009).

\section{Experiment 1}

\section{Method}


Pre-test. Twelve scenarios were formulated involving actions that could in principle be moralized but that would nevertheless be judged morally neutral. In addition, 4 control scenarios were included: two scenarios involving a morally wrong action and two scenarios involving a morally right action. The control scenarios were included to break the pattern and to enable the participants to use the full rating scale. The scenarios were presented to the participants $(N=54)$ in a pseudo-random order, so that the control scenarios were presented at roughly equal intervals. The participants were instructed to "evaluate the morality of the described actions". They rated the scenarios on an 11-point Likert-scale ranging from -5 (morally totally wrong) through 0 (neither right nor wrong) to +5 (morally totally right). The survey was conducted on the Internet, using the web-based survey utility Webropol. Recruitment was conducted through psychology students' mailing list at University of Turku, Finland.

Six of the 12 morally neutral scenarios were chosen for the experiment. The selection criteria were the following: the mean, mode and median of the rating of the scenario were between -1 and +1 . The ratings of the pre-test scenarios are presented in Table 1.

Table 1

Mean ratings for the permissibility of the actions in the pre-test scenarios. The selected scenarios are marked with an asterisk (*). The scale is from -5 (morally totally wrong) to +5 (morally totally right).

\begin{tabular}{lllll}
\hline Scenario & Mean & Median & Mode & SD \\
\hline Neutral & & & & \\
\hline Matti & -2.33 & -2 & -1 & 1.58 \\
Eerik & -1.22 & -1 & -2 & 1.75 \\
Pekka & -1.15 & -1 & -1 & 1.47 \\
Laura* & -0.98 & -1 & -1 & 1.50 \\
Simo* & -0.70 & -1 & -1 & 2.58 \\
Kauko* & -0.57 & -1 & -1 & 1.83
\end{tabular}




\begin{tabular}{lcccc} 
Maj-Lis* & 0.37 & 0 & 0 & 1.57 \\
Maija* & 0.39 & 0 & -1 & 2.06 \\
Sofia* & 0.58 & 0 & 0 & 1.87 \\
Henry & 1.94 & 0 & 0 & 2.26 \\
Mikko & 2.13 & 0 & 0 & 2.44 \\
Esa & 2.41 & 3 & 0 & 2.34 \\
\hline Right & & & & \\
\hline Stefan* & 4.41 & 5 & 5 & 1.07 \\
Elina & 4.70 & 5 & 5 & 0.84 \\
\hline Wrong & & & & \\
\hline Jesse & -4.56 & -5 & -5 & 0.72 \\
Heli* & -4.15 & -5 & -5 & 1.22 \\
\hline
\end{tabular}

The selected morally neutral scenarios were the following: Simo is a married man who secretly flirts with his female colleagues, although he never crosses the line; Sofia gossips to her friends that their 47-year-old male professor is dating a 24-year-old female student; Maj-Lis walks across a charity worker on the street but does not donate because she intends to use her change for a cup of coffee; Laura smokes at her workplace secretly from his husband, who disapproves smoking; Maija reads a magazine addressed to her neighbor, accidentally put in her mailbox by the postman, before returning it; Kauko knows about the carbon dioxide emission of airplanes and their effect on the climate, but nevertheless flies to Tenerife on his summer vacation. The morally wrong control scenario selected for the actual experiment was about Heli, who intentionally gave wrong directions to a group of gypsies who were looking for a spa; the morally right control scenario selected was about Stefan, who saved his neighbor's cat from a tree. 
Participants. The experiment was conducted with university students $(N=87,37$ male) attending three different lectures: educational psychology (University of Turku, Finland, $n=19,2$ male), change management (Turku School of Economics, Finland, $n=37,19$ male), and political history (University of Turku, Finland, $n=31,16$ male). Sample size was not determined a priori, but instead we aimed to gather as many participants as possible. The participants were randomized into an experimental group ( $n=45,19$ male) and control group ( $n=42,18$ male). The participants were warned both on the title sheet and verbally about the possibility of seeing potentially disturbing pictures, and it was emphasized that participating in the experiment was voluntary.

Materials and procedure. Two variants of a paper questionnaire were used, one for the experimental (disgust) group and the other for the control group. The questionnaires were given randomly to the students during the lectures, except for the last test group (political history), in which more experimental questionnaires were given to men to balance the number of males and females in the experimental group.

As a method of emotional induction carried out for the experimental group, the participants were asked to evaluate the interestingness and disgustingness of six colored photographs on a 0-4 scale. The interestingness ratings were included to conceal the experimental purpose of the pictures. The pictures were chosen using Google image search. We did not use standardized image databases such as photographs from the International Affective Picture System (IAPS; Lang \& Bradley 2008), because we judged that the IAPS pictures were either not disgusting enough, or they referred to morally relevant actions (e.g., pictures of victims of violence). In the control group, the pictures were emotionally neutral pictures from the same or higher-level semantic categories as the experimental stimuli. The stimuli are listed in Table 2. 
Stimuli used for emotional induction

\begin{tabular}{lll}
\hline Experimental & Control & Semantic category \\
\hline Fly maggots in dog food & Butterfly & Insect \\
Severe dermatophytosis in nails of the foot & Female feet in sandals & Body part \\
Infected wound in the sole of a foot & Bare male feet on sand & Body part \\
Human faeces in a toilet seat & Clean bathroom sink & Toilet furniture \\
Very bad teeth & Neutral female face & Body part \\
A large neoplastic tumor in armpit & Healthy arm & Body part \\
\hline
\end{tabular}

The experimental stimuli were the six morally neutral scenarios chosen in the pre-test, plus a wrong and right control scenario, making up 8 scenarios altogether. The control scenarios were included to encourage the participants to use the full rating scale. The participants were asked to "evaluate the morality of the action" in the scenarios. A 160-mm visual analogue (VA) scale was used, with the end points titled "morally totally wrong" and "morally totally right", and the middle point (marked with a short vertical line) titled "neither morally right nor wrong".

To measure the participants' political orientation and disgust sensitivity, they were asked to rate their position on the political left-right axis and their position on the liberal-conservative axis on VAscales, and to fill in a disgust sensitivity scale (DS-R, Haidt, McCauley \& Rozin, 1994, modified by Olatunji et al., 2007). The VA-scales for the political questions were $160 \mathrm{~mm}$ long, with the end points titled "left" and "right", and "liberal" and "conservative". The center point of the scale was marked with a short vertical line. The sum score from DS-R was used as a measure of disgust sensitivity.

The effectiveness of the disgust manipulation was tested using ratings of faces depicting Ekman's six basic emotions (joy, surprise, disgust, fear, sorrow, and anger). The participant was asked to rate on a 0-4 Likert scale how much their mood corresponded to each of the photographs. 
Photographs were used because we assumed they would probe the participants' mood more directly and validly than verbal representations.

The order of the sections in the sheet was the following: 1. Political orientation, 2. DS-R, 3. Picture Rating, 4. Moral Rating, and 5. Mood Rating. The emotional induction (and control) pictures were positioned on the same spread with the moral scenarios to maximize possible effect on the scenarios. The Mood Rating appeared on the last page, since the induced emotion should be present throughout the scenarios.

\section{Results}

The experimental and control groups were matched in terms of sex, age, DS, and political orientation (Left-Right and Liberal-Conservative), with threshold levels of $p s \geq .3$. In the initial sample $(N=87)$, participants were more on the political right in the control group than in the experimental group. Thus, we excluded from the control group six participants most positive (i.e., representing the Right) on the Left-Right measure. In the final sample $(n=81)$, the groups did not differ on sex $\left(\chi^{2}=\right.$ $.040, p=.84)$, age $(t=-.086, p=.93)$, DS $(t=.44, p=.67)$, Left-Right scale $(t=-1.05, p=.30)$, or Liberal-Conservative scale $(t=-.70, p=.48)$. All subsequent analyses were performed on the sample with matched groups. The key participant characteristics in the final sample are summarized in Table 3.

The Left-Right rating correlated positively with the Liberal-Conservative rating $(r=.51, p<$ .001 ; i.e., conservatism was associated with being on the political right), but neither the Left-Right nor Liberal-Conservative rating correlated with disgust sensitivity $(r s<.16, p s>.17)$.

Table 3

Key participant characteristics in the matched sample $(n=81)$ 


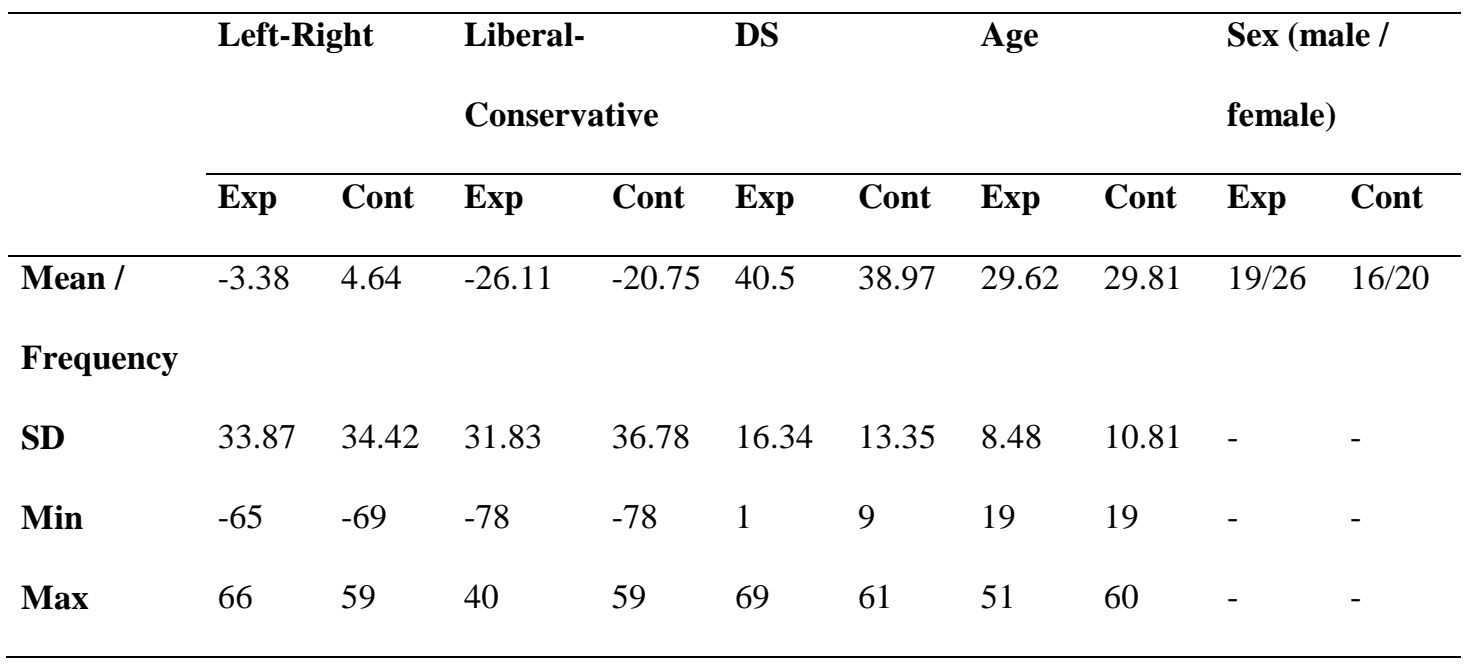

Effectiveness of the emotional induction procedure. The Mood Ratings between the experimental and control group are summarized in Table 4. Both disgust and fear were experienced more in the experimental group ( $p s \leq .05$ ), but disgust showed clearly the largest difference. In terms of Bayes factor $(\mathrm{BF})$, evidence for greater disgust in the experimental group was strong $\left(B F_{10}>10\right)$, but there was only weak evidence for a difference in other emotions $\left(B F_{10}\right.$ 's $\left.<3\right)$.

Table 4

Differences between the experienced affect between the experimental and control group. Rank-Biserial correlation $\left(r_{R B}\right)$ was used as an estimate of standardized effect size.

\begin{tabular}{|c|c|c|c|c|c|c|c|c|c|}
\hline & \multicolumn{2}{|l|}{ Mean (SD) } & \multirow[b]{2}{*}{$\mathbf{U}$} & \multirow[b]{2}{*}{$\mathbf{p}$} & \multirow[b]{2}{*}{ rRB } & \multicolumn{2}{|c|}{$95 \% \mathrm{CI}$ for $\mathrm{r}_{\mathrm{RB}}$} & \multirow[b]{2}{*}{$\eta_{p}^{2}$} & \multirow[b]{2}{*}{$\mathrm{BF}_{10}$} \\
\hline & $\operatorname{Exp}$ & Cont & & & & Lower & Upper & & \\
\hline Happy & $2.53(1.12)$ & $2.47(1.25)$ & 815 & .97 & .006 & -0.24 & .25 & .001 & .24 \\
\hline Surprised & $2.20(.99)$ & $1.69(1.23)$ & 983 & .050 & .248 & -0.00 & .47 & .052 & 1.47 \\
\hline Disgust & $1.16(1.17)$ & $.43(.82)$ & 1084 & .002 & .376 & 0.14 & .57 & .112 & 14.45 \\
\hline Fear & $.96(1.07)$ & $.46(.74)$ & 1003 & .022 & .274 & 0.02 & .49 & .067 & 2.50 \\
\hline Sorrow & $.38(.78)$ & $.40(.70)$ & 766 & .79 & -.028 & -0.28 & .23 & .000 & 0.24 \\
\hline Angry & $.56(.97)$ & $.34(.68)$ & 848 & .46 & .077 & -0.18 & .32 & .015 & 0.40 \\
\hline
\end{tabular}


The experimental pictures were rated as more disgusting $(M=3.01, S D=.80)$ than the control pictures $\left(M=.25, S D=.38 ; U=1491, p<.001, B F_{10}>100\right)$. The experimental pictures were rated as less interesting $(M=.78, S D=.83)$ than the control pictures $(M=1.62, S D=.79 ; U=354, p<.001$, $\left.B F_{10}>100\right)$.

Morality ratings. Morality ratings for each scenario are summarized in Table 5. To test for the overall effect of induced disgust, the sum variable of Mean Morality was computed for the ratings of the six neutral scenarios. Mean Morality did not differ between the experimental group $(M=3.56, S D=$ $17.11)$ and the control group $\left(M=3.17, S D=13.42 ; W=766, p=.68, \eta_{p}{ }^{2}=.00, B F_{10}=.23\right)$. The inverted Bayes Factor was $B F_{01}=4.28$, indicating substantial evidence for the null hypothesis. ${ }^{2}$

Table 5

Morality ratings for all scenarios in the experimental and control conditions. Positive values represent morally right and negative morally wrong judgments.

Meutral
Simo
The Bayes Factor is an estimate for how many more times plausible the data is under the alternative hypothesis compared
to the null hypothesis. BF above 1 indicates evidence for the alternative hypothesis, whereas BF below 1 indicates
evidence for the null hypothesis. The inverted BF was used as a more intuitive measure of evidence for the null effect,
because the values of the inverted BF increase as the null hypothesis receives more support. BF between 1 and 3 can be
considered as weak evidence for the alternative hypothesis, $3-10$ substantial, and 10-30 strong. Respective values apply
with respect to the inverted BF and the null hypothesis (Jeffreys, 1961).




\begin{tabular}{lll} 
Sofia & $3.53(23.90)$ & $8.08(22.02)$ \\
MajLis & $13.69(30.99)$ & $15.14(31.68)$ \\
Laura & $-6.80(32.17)$ & $-10.58(24.05)$ \\
Maija & $0.11(31.32)$ & $0.39(32.85)$ \\
Kauko & $9.38(35.37)$ & $6.11(29.06)$ \\
Mean Morality & $3.56(17.11)$ & $3.17(13.42)$ \\
Wrong & & \\
Heli & $-56.8(25.59)$ & $-59.69(24.61)$ \\
Right & & \\
Stefan & $67.49(18.69)$ & $71.86(9.37)$ \\
\hline
\end{tabular}

To test for the possible mediating effects of the participants' Disgust Sensitivity (DS) and their position on the Liberal-Conservative (LibCon) and Left-Right (LeftRight) scales on Mean Morality, an ANCOVA was performed with Mean Morality as the dependent variable, Group as the fixed factor, and DS, LibCon, and LeftRight as the covariates. To test for possible moderating effects, the model included interactions between Group and each of the covariates. Group did not interact with any of the covariates $(F \mathrm{~s}(1,65)<2, p \mathrm{~s}>.16)$, and none of the main effects were significant $(F \mathrm{~s}(1,65)<2.2, p$ 's $>$ $.14)$, except for the main effect of LeftRight $(F(1,65)=4.34, p=.041$; the more a participant was on the political right, the more they considered the actions as morally correct). Bayesian analyses did not support the alternative hypothesis, but instead there was strong evidence for the lack of interaction between Group and LibCon or DS $\left(B F_{01} \mathrm{~S}>15\right)$, and substantial evidence for the lack of interaction between Group and LeftRight $\left(B F_{01}=3.91\right)$. Likewise, there was substantial evidence for the lack of main effect of Group, LibCon, and DS $\left(B F_{01} \mathrm{~s}>3.70\right)$, but no evidence for or against main effect of LeftRight $\left(B F_{10}=.98\right)$. 


\section{Discussion of Experiment 1}

Experiment 1 examined the effect of induced disgust on morally neutral scenarios to test the moralization hypothesis, which implies that disgust can moralize an action. The disgust induction procedure was successful, as the participants in the experimental group reported experiencing more disgust than participants in the control condition. Despite this, there were no differences in aggregated moral judgments between the experimental and control groups, even after controlling for possible interactions with the participants' disgust sensitivity and political orientation. On the contrast, the Bayesian analyses provided substantial support for the null hypothesis that disgust does not affect moral ratings, and strong-to-moderate evidence for the lack of interaction between Group and the background variables. These findings are evidence against the moralization hypothesis, but they do not lend support for any specific hypothesis; instead, they are compatible with the amplification hypothesis or even a purely rationalist account of moral judgment. On the other hand, the sample size in Experiment 1 was relatively small, especially given that the effect size is supposedly minimal. To more directly test whether disgust has a differential effect on neutral and wrong moral scenarios, we conducted Experiment 2 which included both neutral and wrong scenarios and a larger sample.

\section{Experiment 2}

\section{Method}

Participants. We aimed to gather at least 500 participants, which enables discovering a small effect $(d=.25)$ with .05 alpha level and power of .80 . The participants were recruited through e-mail lists and Facebook advertising. As a cover story we said that the experiment was about associations between visual attention and reading comprehension. Because deceit was used, we sought for ethical approval of the experiment from the research ethics committee at Abo Akademi University, Finland. The participants were warned about the possibility of seeing disturbing material during the experiment. 
When the participants clicked on the recruiting link, they were randomly redirected to a Google Form that included the moral scenarios and either disgusting or emotionally neutral videos. Altogether 510 people responded (268 in the experimental group). However, $451(88 \%)$ of the respondents were female, which made it impossible to generalize across the whole tested population. Thus, we decided to exclude all non-women. Additionally, two participants were excluded because all their moral ratings were "1", which we considered as a random answer pattern. Thus, the final sample was $n=448$.

Materials and procedure. The participants first filled in a background questionnaire, which probed their gender, age, education, which political party best represented their values, and net income. DS was not included in this experiment, because we had double the number of moral scenarios compared to Experiment 1, and wanted to keep the experiment short to maximize the number of participants. The moral scenarios were the same as in Experiment 1, with the addition of morally wrong variants of each, which were the following: Simo flirts with his female colleagues and during one evening after the workplace's pre-Christmas party, he passionately kisses with his female colleague and does not tell his wife about this (in the neutral version Simo never crosses the line); Sofia posts gossips about her professor in a Facebook group, from where the rumors start to spread (in the neutral version Sofia privately gossips about the professor); Maj-Lis walks across a charity worker on the street and angrily shouts at him that he should spend his time more wisely (in the neutral version Maj-Lis passively passed the worker); Laura smokes secretly at her workplace and lies to her husband that she hasn't smoked when he wonders why her clothes smell (in the neutral version the husband doesn't ask and Laura doesn't tell); Maija takes the magazine addressed to her neighbor and doesn't return it (in the neutral version she does return it); Kauko flies to Thailand each month because he can afford that (in the neutral version he flies to Tenerife on his summer vacation). The neutral scenarios and their wrong variants totaled 12 scenarios (6+6). In addition, we included two extremely wrong scenarios to avoid a ceiling effect in the evaluations of the moderately wrong actions. The extremely wrong scenarios were 
the following: Pekka becomes violent when he uses alcohol, and once during drinking he hits a stranger who falls, hits his head and dies; Anu hurries to work by car, simultaneously reading e-mail on her cell phone, when she hits a child on crosswalk, killing the child. The extremely wrong scenarios were always presented as first and in the middle of the other scenarios, which were counterbalanced in order. The scenarios were evaluated on a seven-point Likert scale ( $1=$ "completely wrong"; 7 = "completely right").

For emotional induction, we used film clips validated by Hewig et al. (2005). For disgust induction, two film clips were used: A scene from the movie Pink Flamingos (dir. John Waters) where a dog defecates on the street and a person picks up the feces and eats them; and The horse head scene from The Godfather (dir. Francis Ford Coppola), where a person finds the decapitated head of a horse in his bed and starts screaming. In the neutral emotion group the following clips were used: an excerpt from Hannah And Her Sisters (dir. Woody Allen) depicting discussions between different people; and a scene from All The President's Men (dir. Alan J. Pakula), depicting two journalists trying to crack the Watergate conspiracy. The films were presented in counterbalanced order, one before any of the scenarios and the other in the middle of the scenarios. The participants evaluated how arousing and disgusting the videos were on a Likert scale from 1 (not at all disgusting/arousing) to 5 (extremely disgusting/arousing). The question about arousal was included to conceal the purpose of the study.

After all the moral scenarios, the participants evaluated how strongly they felt joy, surprise, disgust, fear, sorrow, and anger on a scale from 1 (don't feel at all) to 5 (feel very strongly). Finally, the purpose of the study was revealed to them and they got to answer whether they had guessed its purpose.

\section{Results}


Political orientation on the left-right (LeftRight) and liberal-conservative (LibCon) axes were coded based on Kivikangas' (2017) analysis of politicians' answers to questions about values in the communal elections test ("match your vote") of Helsingin Sanomat (the main newspaper in Finland). The politicians were grouped based on which party they belonged to, and their mean answers determined the party's overall mean in LeftRight and LibCon.

As in Experiment 1, the experimental and control groups were matched in terms of age, education, income level, LeftRight, and LibCon with threshold levels of $p s \geq .3$ (sex was not matched because only females were included). Average income was higher $(U=34324, p=.24)$ in the control group than in the experimental group. Thus, we removed four participants with highest wage in the control group plus three participants with the lowest income in the experimental group, resulting in a final sample of $n=441$ (228 in the experimental and 213 in the control group). In the matched sample, the groups did not differ with respect to age $(t=.22, p=.83)$, education $(U=24679, p=.75)$, income $(U=25553, p=.32)$, LeftRight $(U=17033, p=.39)$, or LibCon $(U=17365, p=.59)$. Key participant characteristics in the final sample are summarized in Table 6.

Table 6.

Key participant characteristics by Group in Experiment 2.

\begin{tabular}{llllllllllll}
\hline & Left-Right & \multicolumn{3}{c}{$\begin{array}{l}\text { Liberal- } \\
\text { Conservative }\end{array}$} & \multicolumn{3}{c}{ Age } & & \multicolumn{2}{c}{ Education } & \multicolumn{3}{c}{ Income } \\
\cline { 2 - 12 } & Exp & Cont & Exp & Cont & Exp & Cont & Exp & Cont & Exp & Cont \\
\hline Mean & 2.26 & 2.19 & 2.32 & 2.28 & 34.91 & 35.15 & 2.98 & 3.01 & 2.04 & 2.14 \\
SD & .66 & .60 & .67 & .63 & 12.33 & 11.39 & .88 & .88 & .93 & .98 \\
Min & 1.46 & 1.46 & 1.84 & 1.84 & 18 & 18 & 1 & 1 & 1 & 1 \\
Max & 3.67 & 3.67 & 4.15 & 4.15 & 71 & 71 & 4 & 4 & 5 & 5 \\
\hline
\end{tabular}

LibCon and LeftRight correlated positively $(r=.65, p<.001)$, that is, participants who were on the political right were also more conservative. Income and education also correlated positively $(r=$ $.24, p<.001)$, as well as Age and Income $(r=.42, p<.001)$. Other associations between the 
background variables were not significant. At the end of the study, 148 (33.6\%) of the participants reported having guessed its purpose.

Effectiveness of the emotional induction procedure. Mood ratings in the experimental and control groups are summarized in Table 7. In terms of p-values, happiness, surprise, and disgust were experienced more in the experimental than in the control group, but the effect size was by far largest for disgust. Bayes Factors indicated evidence only for a difference in disgust $\left(B F_{10}>100\right)$ but not in the other emotions $\left(B F_{10 \mathrm{~s}}<2.3\right)$.

Table 7

Self-reported mood at the end of Experiment 2.

\begin{tabular}{|c|c|c|c|c|c|c|c|c|c|}
\hline & \multicolumn{2}{|l|}{ Mean (SD) } & \multirow[b]{2}{*}{$\mathbf{W}$} & \multirow[b]{2}{*}{$\mathbf{p}$} & \multirow[b]{2}{*}{$\mathbf{r}_{\mathbf{R B}}$} & \multicolumn{2}{|c|}{ 95\% CI for $r_{R B}$} & \multirow[b]{2}{*}{$\eta_{p}^{2}$} & \multirow[b]{2}{*}{$\mathbf{B F}_{10}$} \\
\hline & $\operatorname{Exp}$ & Cont & & & & Lower & Upper & & \\
\hline Happy & $2.50(1.07)$ & $2.70(1.08)$ & 26828 & .048 & .091 & -.003 & .18 & .008 & .61 \\
\hline Surprised & $2.17(1.16)$ & $1.90(1.09)$ & 20962 & .009 & -.12 & -.21 & -.026 & .014 & 2.20 \\
\hline Disgust & $2.13(1.20)$ & $1.37(.79)$ & 15084 & $<.001$ & -.35 & -.43 & -.26 & .12 & $>100$ \\
\hline Fear & $1.66(.94)$ & $1.59(.98)$ & 22820 & .21 & -.037 & -.13 & .056 & .001 & .14 \\
\hline Sorrow & $2.00(1.14)$ & $1.90(1.11)$ & 22988 & .30 & -.046 & -.14 & .048 & .002 & .17 \\
\hline Angry & $1.56(.89)$ & $1.41(.78)$ & 22317 & .071 & -.083 & -.18 & .011 & .007 & .46 \\
\hline
\end{tabular}

The experimental videos were rated as more disgusting than the control videos $(M=3.78, S D=$ .86 VS. $\left.M=1.50, S D=.68 ; U=1590, p<.001, B F_{10}>100\right)$, as well as more arousing $(M=2.65, S D=$ .90 VS. $\left.M=2.15, S D=.73 ; U=16322, p<.001, B F_{10}>100\right)$.

Morality ratings. Before examining the morality ratings in detail, we checked whether guessing the purpose of the study was associated with the moral ratings. Thus, we conducted one-way ANOVA with average moral rating (average from neutral and wrong scenarios) as dependent variable and Group (experimental/control) and Guessed (yes/no) as between-subjects factors. There was no main effect of Group $\left(F<.001, p>.99, B F_{01}=8.91\right)$ or Guessed $\left(F=.74, p=.39, B F_{01}=6.44\right)$, or 
their interaction $\left(F=.33, p=.57, B F_{01}=5.52\right.$ [null model included main effects of Group and Guessed]). In terms of Bayes factors, there was substantial evidence for the null hypothesis that guessing the purpose of the study did not affect the results; thus, we proceeded to the main analysis without further exclusions.

Morality ratings in each of the scenarios and means for the neutral and wrong scenarios per group are summarized in Table 8. Main effects of Scenario Type (neutral vs. wrong), Group, and their interaction were examined in an ANOVA with the mean morality rating as the dependent variable, Scenario Type as the within-subjects factor, and Group as the between-subjects factor. There was no main effect of Group $\left(F=.13, p=.72, \eta_{p}{ }^{2}=.00, B F_{01}=12\right)$, but a significant effect of Scenario Type $\left(F=896, p<.001, \eta_{p}{ }^{2}=.67, B F_{10}>100\right)$. There was no interaction between Group and Scenario Type $\left(F=.086, p=.77, \eta_{p}{ }^{2}=.00, B F_{01}=8.77\right.$ [null model included main effects of Group and Scenario Type]). That is, in terms of the Bayes Factors, there was substantial evidence for the null hypothesis that disgust does not affect moral ratings.

Table 8 .

Ratings of the individual scenarios and means by Group

\begin{tabular}{lll}
\hline & \multicolumn{2}{l}{ M (SD) } \\
\cline { 2 - 3 } Neutral & & \\
\cline { 2 - 3 } Simo & & \\
Sofia & $3.18(1.40)$ & $3.18(1.42)$ \\
MajLis & $4.88(1.30)$ & $3.91(1.42)$ \\
Laura & $3.78(1.61)$ & $3.85(1.30)$ \\
Maija & $3.37(1.55)$ & $3.38(1.39)$ \\
Kauko & $4.08(1.54)$ & $3.90(1.43)$ \\
Mean Neutral & $3.84(1.12)$ & $3.83(.97)$ \\
Wrong & & \\
Simo & $1.66(.89)$ & $1.87(1.20)$ \\
Sofia & $1.93(1.02)$ & $1.56(.77)$ \\
MajLis & $1.78(.92)$ & $1.93(1.14)$
\end{tabular}




\begin{tabular}{lll} 
Laura & $2.91(1.30)$ & $2.78(1.33)$ \\
Maija & $1.85(1.26)$ & $1.70(.93)$ \\
Kauko & $2.71(1.49)$ & $2.75(1.38)$ \\
Mean Wrong & $2.14(.78)$ & $2.10(.74)$ \\
Extremely wrong & & \\
Pekka & $1.09(.48)$ & $1.02(.15)$ \\
Anu & $1.17(.51)$ & $1.17(.41)$ \\
\hline
\end{tabular}

Next, we examined whether the background variables moderated the effect of group. We used an ANCOVA with Group as the between-subjects factor, Scenario Type as the within-subjects factor, and age, income, education, LeftRight, and LibCon as the covariates. In this model, there was again no main effect of Group $\left(F=.24, p=.62, \eta_{p}{ }^{2}=.00, B F_{01}=10\right)$, but there was a main effect of Scenario Type $\left(F=21.75, p<.001, \eta_{p}^{2}=.055, B F_{10}>100\right)$. There was no interaction between Group and Scenario Type $\left(F=.082, p=.78, \eta_{p}{ }^{2}=.00, B F_{01}=7.98\right.$ [null model included main effects of Group and Scenario Type]). None of covariates predicted the moral ratings $\left(p s>.06, \mathrm{BF}_{10}<1.2\right)$, and neither did they interact with Group $\left(p \mathrm{~s}>.06, B F_{0 I} \mathrm{~s}>1.80\right.$ [each Covariate $\times$ Group interaction was added individually and tested against null model that included the main effects of all the Covariates, Group, and Scenario Type]).

\section{Discussion of Experiment 2}

In Experiment 2 we aimed to test the differential effect of disgust on neutral and wrong moral scenarios. The amplification hypothesis predicts that accidental disgust makes judgments of morally wrong actions more severe but does not affect the neutral scenarios; the moralization hypothesis is a stronger theory that predicts that disgust can make otherwise morally neutral scenarios condemnable. The emotional induction was successful, as participants in the experimental group felt substantially more disgusted than participants in the control group, with no remarkable differences in other 
emotions. Despite this, there was neither a main effect of induced disgust, nor interaction between disgust induction and type of scenario. In contrast, Bayes Factors provided substantial evidence against the effect of disgust. These findings are in conflict both with the stronger moralization hypothesis and the weaker amplification hypothesis.

\section{General Discussion}

The moralization hypothesis (that disgust can moralize otherwise neutral actions) has not been systematically tested before, because previous studies have mainly used as stimuli scenarios that are judged to be morally wrong even without any emotional induction. We tested the moralization hypothesis in two experiments that involved morally neutral scenarios, that is, scenarios that are judged as morally neither wrong nor right when no emotional induction is used. Additionally, we tested the differential effect of disgust on neutral and wrong variants of the same scenarios. The results of Experiment 1 provided substantial evidence against the hypothesis that disgust would moralize neutral actions. Experiment 2 provided substantial evidence both against the moralization hypothesis and the amplifying hypothesis: disgust did not moralize neutral actions, and neither did it amplify morally wrong scenarios. These results are in line with the meta-analysis by Landy and Goodwin (2015), which indicated that accidental disgust does not make moral judgments more severe. On the other hand, Landy and Goodwin found some evidence for the moralization hypothesis, which is surprising, given that the moralization hypothesis is stronger than the amplification hypothesis: if the latter is incorrect, it is very improbable that the former would be true. However, previous evidence for the moralization hypothesis is mainly anecdotal (individual scenarios from individual studies; e.g., Wheatley \& Haidt, 2005), whereas the present study was the first where it was systematically tested with large samples.

Even though accidental disgust does not affect moral ratings, this does not imply that emotion would not have any role in moral judgment. It is possible that emotion elicited by the stimulus itself can 
affect its moral evaluation; for example, a depiction of the married man Simo kissing with his female colleagues can elicit more negative emotions than a depiction of him simply flirting with his colleagues, leading to the moralization of the former action. However, this is mere speculation. The hypothesis that negative emotion could moralize an action is mainly supported by the correlational studies where moral judgment was found to be associated mainly with emotions, not ratings of harm (e.g., Haidt, Koller, \& Dias, 1993; Haidt \& Hersh, 2001). Such findings can be interpreted both in line with the moralization and the elicitation hypothesis: it might be that emotions do not cause moral judgments, but instead that, for example, conceptually made moral judgments bring about emotions. Experimental studies are needed to determine the direction of causality, but the problem is how to artificially manipulate which emotions an action itself produces. One possibility could be to manipulate, for example, the appearance of the person conducting the action that is evaluated.

All in all, our results are more in line with the classical rationalist, harm-based theory of moral judgment (e.g., Turiel, 1983; Royzman, Leeman, \& Baron, 2009) than moral emotivism. In our experiments, scenario type had a very large effect size - a trivial finding that can be taken to indicate that what mainly affects moral judgment are the conceptual processes related to the interpretation of the scenario. Of course, it is possible that some emotional processes that occur after conceptually processing the scenario affect the moral ratings, but there is very little experimental support for this hypothesis.

\section{Limitations and Strengths}

One could argue that the disgust induction was too weak to influence the aggregated moral judgments. However, the standardized effect sizes (partial eta squared .11 in Experiment 1 and .12 in Experiment 2) were similar to those in previous studies. For instance, in the Schnall et al. (2008) study, effect size was .19 in Experiment 1, non-significant in Experiment 2 (effect size not reported), and .06 
in Experiment 3, but they nevertheless found an amplifying effect of disgust on aggregated moral ratings in each of the experiments. Another possible limitation of the current study is that making strong conclusions based on null effects can be problematic, because absence of evidence is not typically evidence of absence. To counter this problem, we used Bayesian statistics, which provides a continuous measure of evidence for the alternative or the null hypothesis.

The main strength of the current study is that it utilized for the first time pre-tested neutral moral scenarios, which enabled to directly and systematically test the moralization hypothesis. Previous studies have mainly used morally wrong actions, which makes it impossible for them to directly test the moralization hypothesis. Additionally, a strength of Experiment 2 is that it used neutral and wrong variants of the same moral scenarios. This reduces random variance due to the scenario formulations, and enables to more directly test the differential effect of disgust on morally neutral and wrong behavior.

\section{Conclusions}

Earlier studies on the effect of disgust on moral ratings have used as stimuli actions that are judged to be wrong even without any emotional manipulation. Thus, they cannot test whether disgust is sufficient to moralize an action, but instead can only test the amplification hypothesis. In the present study, we tested the moralization hypothesis by examining the effect of artificially induced disgust on neutral moral scenarios, as well as the differential effect of disgust on neutral and wrong scenarios. The results did not support the moralization or the amplification hypotheses. Instead, Bayesian analysis lent substantial evidence for the null hypothesis that disgust has no effect on moral judgment. The results are consistent with the meta-analysis of Landy and Goodwin (2015) and in line with a rationalist, harmbased theory of moral judgment. 


\section{References}

Avramova, Y. R., \& Inbar, J. (2013). Emotion and moral judgment. WIREs Cognitive Science, 4, 169178.

Cannon, P. R., Schnall, S., \& White, M. (2011). Transgressions and expressions: Affective facial muscle activity predicts moral judgments. Social Psychological and Personality Science, 2, 325331.

Chapman, H. A., \& Anderson, A. K. (2014). Trait physical disgust is related to moral judgments outside of the purity domain. Emotion, 14, 341-348.

Chapman, H. A., Kim, D. A., Susskind, J. M., \& Anderson, A. K. (2009). In bad taste: Evidence for the oral origins of moral disgust . Science, 323, 1222-1226.

Eskine, K. J., Kacinik, N. A., \& Prinz, J. J. (2011). A bad taste in the mouth: Gustatory disgust influences moral judgment. Psychological Science, 22, 295-299.

Haidt, J. (2001). The emotional dog and its rational tail: A social intuitionist approach to moral judgment. Psychological Review, 108, 814-834.

Haidt, J., \& Hersh, M. A. (2001). Sexual morality: The cultures and emotions of conservatives and liberals. Journal of Applied Psychology, 31, 191-221.

Haidt, J., Koller, S. H., \& Dias, M. G. (1993). Affect, culture and morality, or is it wrong to eat your dog? Journal of Personality and Social Psychology, 65, 613-628.

Haidt, J., McCauley, C., \& Rozin, P. (1994). Individual differences in sensitivity to disgust: A scale sampling seven domains of disgust elicitors. Personality and Individual Differences, 16, 701-713.

Hewig, J., Hagemann, D., Seifert, J., Gollwitzer, M., Naumann, E. \& Bartussek, D. (2005). A revised film set for the induction of basic emotions. Cognition and Emotion, 19, 1095-1109.

Inbar, J., Pizarro, D. A., \& Bloom, P. (2011). Disgusting smells cause decreased liking of gay men. Emotion, 1-5. 
Inbar, Y., \& Pizarro, D. A. (2009). Conservatives are more easily disgusted than liberals. Cognition and Emotion, 23, 714-725.

Inbar, Y., Pizarro, D. A., Knobe, J., \& Bloom, P. (2009). Disgust sensitivity predicts intuitive disapproval of gays. Emotion, 9, 435-439.

Jeffreys, H. (1961). Theory of Probability. Oxford: Clarendon Press.

Jones, A., \& Fitness, J. (2008). Moral hypervigilance: The influence of disgust sensitivity in the moral domain. Emotion, 8, 613-627.

Kivikangas, M. (2017). Kuntavaalikoneiden (Yle \& HS) arvokysymysten vertailua. Retrieved February $18^{\text {th }} 2019$ from https://jmatiaskivikangas.net/2017/05/05/kuntavaalikoneiden-yle-hsarvokysymysten-vertailua/

Kohlberg, L. (1981). Essays on moral development, Vol. I: The philosophy of moral development. San Francisco, CA: Harper \& Row.

Landy, J. F. \& Goodwin, G. P. (2015). Does incidental disgust amplify moral judgment? A metaanalytic review of experimental evidence. Perspectives on Psychological Science, 10, 518-536.

Landy, J. F., \& Piazza, J. (2019). Reevaluating moral disgust: Sensitivity to many affective states predicts extremity in many evaluative judgments. Social Psychological and Personality Science, 10(2), 211-219. https://doi.org/10.1177/1948550617736110

Lang, P. J., Bradley, M. M., \& Cuthbert, B. N. (2008). International affective picture system (IAPS): Affective ratings of pictures and instruction manual. Technical Report A-8. University of Florida, Gainesville, FL.

Nichols, S. (2002). Norms with feeling: Towards a psychological account of moral judgment. Cognition, 84, 221-236.

Olatunji, B. O., Puncochar, B. D., \& Cox, R. (2016). Effects of experienced disgust on morally-relevant judgments. PLoS One, 11, PMC4970715. 
Olatunji, B. O., Williams, N. L., Tolin, D. F., Abramowitz, J. S., Sawchuk, C. N., Lohr, J. M., et al. (2007). The Disgust Scale: Item analysis, factor structure, and suggestions for refinement. Psychological Assessment, 19, 281-297.

Piaget, J. (1997). The moral judgement of the child. Glencoe, IL: Free Press.

Piazza, J., \& Landy, J. F. (2019). Folk beliefs about the relationships anger and disgust have with moral disapproval. Cognition and Emotion. https://doi.org/10.1080/02699931.2019.1605977

Pizarro, D., Inbar, Y., \& Helion, C. (2011). On disgust and moral jugdement. Emotion Review, 3, 267268.

Prinz, J. (2006). The emotional basis of moral judgements. Philosophical Explorations, 9, 29-43.

Royzman, E. B., Leeman, R. F., \& Baron, J. (2009). Unsentimental ethics: Towards a content-specific account of the moral-conventional distinction. Cognition, 112, 159-174.

Schnall, S., Haidt, J., Clore, G. L., \& Jordan, A. H. (2008). Disgust as embodied moral judgment. Personality and Social Psychology, 34, 1096-1109.

Turiel, E. (1983). The development of social knowledge: Morality and convention. Cambridge, England: Cambridge University Press.

Tracy, J. L., Steckler, C. M., \& Heltzel, G. (2019). The physiological basis of psychological disgust and moral judgments. Journal of Personality and Social Psychology, 116(1), 15-32. https://doi.org/10.1037/pspa0000141

Ugazio, G., Lamm, C., \& Singer, T. (2012). The role of emotions for moral judgments depends on the type of emotion and moral scenario. Emotion, 12, 579-590.

Wheatley, T., \& Haidt, J. (2005). Hypnotic disgust makes moral judgments more severe. Psychological Science, 16, 780-784. 
Wike, R., Stokes, B., \& Simmons, K. (2016). Europeans fear wave of refugees will mean more terrorism, fewer jobs. Retrieved from Pew Research Center website: http://www.pewglobal.org/2016/07/11/negative-views-of-minorities-refugees-common-in-eu/ 